\title{
0 impacto dos maus-tratos na infância no desenvolvimento cerebral e no funcionamento cognitivo: uma revisão
}

\author{
The impact of childhood maltreatment on brain \\ development and cognitive functioning: a review
}

\section{Katiúscia Nunes', Lauren Tonietto ${ }^{2}$, Kelen Dias ${ }^{3}$ e Carolina Rego}

\begin{abstract}
Resumo: $O$ presente artigo teve por objetivo realizar uma revisão na literatura a fim de compreender o impacto dos maus-tratos na infância no desenvolvimento cerebral e no funcionamento cognitivo. Tal pesquisa se mostra importante, tendo em vista a prevalência de crianças que sofrem maustratos e enfrentam adversidades na infância, além do significativo número de adultos e idosos que referem terem sido expostos a traumas na infância. Foram selecionados artigos em língua Portuguesa Brasileira e Inglesa dos últimos 5 anos (de 2015 a 2020) nas principais bases de dados: Pubmed ( $n=358)$, Scielo $(n=2)$ e Google Acadêmico $(n=278)$. Foram incluídos no presente estudo 23 artigos. Os resultados encontrados apontam para alterações nas estruturas cerebrais, principalmente envolvendo sistema límbico, hipocampo e amígdala, córtex pré-frontal e corpo caloso, e alterações na funcionalidade cognitiva, que envolve prejuízos nas funções executivas, nas memórias, na velocidade de processamento e na aprendizagem. Portanto, de acordo com os dados da literatura, os maus-tratos na infância impactam de forma significativa e prolongada as estruturas cerebrais, assim como o funcionamento cognitivo dos indivíduos, perpetuando ao longo da vida. E tendo em vista o impacto social, físico, cognitivo e psicológico que os maus-tratos causam, é indispensável a atenção
\end{abstract}

na sua prevenção, detecção precoce e intervenção, pois seus danos podem se manter por toda vida.

Palavras-chave: Maus-tratos na infância; Desenvolvimento cerebral; Cognição.

\begin{abstract}
This article aimed to conduct a literature review in order to understand the impact of childhood maltreatment on brain development and cognitive functioning. Such research proves to be important, given the prevalence of children who suffer abuse and face adversity in childhood, in addition to the significant number of adults and the elderly who report having been exposed to childhood trauma. Brazilian Portuguese and English articles were selected in the main databases: Pubmed $(n=358)$, Scielo $(n=2)$ and google Scholar $(n=278)$ from the last 5 years (from 2015 to 2020), 23 articles were included in the present study. The results found point to changes in brain structures, mainly involving the limbic system, hippocampus and amygdala, prefrontal cortex and corpus callosum, and changes in cognitive functionality, wich involves impairments in executive function, memories, processing speed and learning. Therefore, according to the literature data, childhood maltreatment has a significant and prolonged impact on brain structures, as well as on individuals'cognitive
\end{abstract}

\footnotetext{
1 Psicóloga. Especialista em Neuropsicologia. Membro efetivo e coordenadora do comitê de neuropsicologia da SPRGS.

E-mail:k.gomes.nunes@gmail.com

2 Psicóloga. Membro efetivo e participante do comitê de neuropsicologia da SPRGS. E-mail: laurentonietto@gmail.com

3 Estudante de Psicologia. Estagiária e participante do comitê de neuropsicologia. E-mail: kelenafdias@gmail.com

${ }^{4}$ Estudante de Psicologia. Estagiária e participante do comitê de neuropsicologia. E-mail: carolina_castanheira@hotmail.com
} 
functioning, perpetuating throughout life. And in view of social, physical, cognitive and psychological impact that mistreatment causes, it is essential to pay attention to its prevention, early detection and intervention, as

\section{Introdução}

Os maus-tratos na infância são fatores de risco para desfechos negativos ao longo da vida, e em torno de 30-40\% da população adulta relata ter experienciado alguma forma de adversidade na infância (Agorastos, Pervanidou, \& Chrousos, 2019). Dentre esses desfechos adversos observa-se: desenvolvimento de psicopatologias, baixo desempenho acadêmico, prejuízos em relacionamentos interpessoais, além de dificuldades no desenvolvimento neuronal e nas habilidades cognitivas (Puetz et al., 2017).

Existem alguns termos que se relacionam com o conceito de maus-tratos. 0 termo Estresse Precoce (EP) descreve um espectro de experiências adversas e estressoras (maus-tratos, negligência, separação, perda dos pais, pobreza, inanição, violência doméstica/comunitária/escolar) desde o primeiro mês de vida até a adolescência. Já 0 termo Trauma na infância representa uma forma mais específica de EP e é definido como "evento traumático que causa ferimento, morte ou afeta a integridade de si ou de outros, e causa horror, terror, ou desamparo no momento que ocorre e oprime a habilidade da pessoa em lidar com a situação (abuso físico/sexual, trauma médico, acidente de carro, terrorismo, experiências de guerra, desastres naturais, testemunha de homicídio ou suicídio)" (Agorastos et al., 2019).

Os maus-tratos são tipos de violência ou falta de cuidado com uma pessoa próxima (geralmente crianças) que estão sob sua custódia, associado a uma forma de agressão no âmbito de uma relação entre duas ou mais pessoas. As formas mais comuns de maus-tratos são as negligências, as violências domésticas e os abusos (físicos, psicológicos, emocionais e sexuais). Os maus tratos emocionais e psicológicos delimitam-se na privação de um ambiente de tranquilidade e de bem-estar afetivo indispensável ao crescimento, desenvolvimento e aos comportamentos equilibrados da criança ou do jovem (Bath, Manzano-Nieves, \& Goodwill, 2016). Os abusos sexuais culminam no envolvimento sexual com um adulto e principalmente, um responsável. A negligência física é a incapacidade de proporcionar à criança ou ao jovem a satisfação de suas necessidades básicas; os abusos ou maus tratos físicos são uma ação não acidental e programada, isolada ou repetida, infligida por pais, cuidadores ou outros com responsabilidade pela criança ou jovem, a qual provoque (ou possa vir a provocar) dano físico; e as violências domésticas são praticadas no âmbito domiciliar e podem ter todos esses conjuntos de maus tratos (Puetz et al., 2017).

Os maus-tratos na infância não provocam apenas traumas psicológicos reversíveis, mas, além disso, danos permanentes no desenvolvimento social, cognitivo, psíquico e em certas funções cerebrais (Puetz et al., 2017). A exposição excessiva ao estresse, especialmente nas fases precoces do neurodesenvolvimento, com maior sensibilidade e maior plasticidade cerebral (primeira infância), pode provocar alteraçōes no estado homeodinâmico da resposta ao estresse e debilitar o desenvolvimento fisiológico (McCrory, Gerin, \& Viding, 2017).

A maioria dos eventos traumáticos, ficam registrados no cérebro, marcando vidas, especialmente no registro de nossas memórias, e na região its damage can be maintained for a lifetime.

Keywords: Childhood maltreatment; Brain development; Cognition.

subcortical, e quando recorrentes, esses eventos têm o potencial de causar alterações cerebrais significativas. Essas costumam ser duradouras e por vezes irreversiveis, ou difíceis de serem moldadas novamente na estrutura e/ou funcionamento da mente, o que se constitui em um fator de risco para o desenvolvimento de prejuízos cognitivos posteriores (Welsh, Peterson, \& Jameson, 2017). A exposição ao estresse nos períodos pré-natais e infantis tem impacto nas estruturas cerebrais envolvidas na cognição e na saúde mental (Bath et al., 2016). Um estudo realizado para comparar o desenvolvimento cerebral em pessoas vitimas de violência e grupo controle, observou que, os hemisférios esquerdos das pessoas vitimadas pela violência desenvolvem-se menos que o de pessoas que não sofreram violência (Thomason \& Marusak, 2017).

0 mecanismo neurobiológico do estresse é caracterizado pela ativação do sistema nervoso autônomo e do eixo hipotálamo-pituitária-adrenal (HPA), que desencadeia respostas neurobiológicas e neuroquímicas, como a liberação de adrenalina e cortisol (Agorastos et al., 2019). Algumas regiões cerebrais como o hipocampo, a amígdala, corpo caloso e o córtex pré-frontal apresentam alterações morfológicas e químicas em resposta ao estresse, que são reversíveis se o estresse não durar excessivamente. No entanto, não se pode garantir a reversibilidade desses efeitos se o estresse for prolongado por períodos maiores (Ershov et al., 2018; Harms, Shannon Bowen, Hanson, \& Pollak, 2018).

0 sistema límbico é uma área situada na parte central do cérebro, para onde confluem os neurônios encarregados de regular a memória e as emoções. Há duas estruturas cruciais para o funcionamento do sistema límbico: o hipocampo e a amígdala. 0 hipocampo é fundamental para a formação e arquivamento das memórias emocionais e verbais. Ea amígdala é responsável pela criação do conteúdo emocional das memórias, associadas às respostas agressivas e ao medo (Teicher \& Samson1., 2016). A vulnerabilidade do hipocampo aos efeitos da negligência ou da violência sofrida pode ser explicada pelo efeito de hormônios como o cortisol, liberados durante o estresse. Dada à possibilidade de 0 ambiente influenciar 0 sistema nervoso central (SNC), é possivel prever que as funções exercidas pelas estruturas encefálicas também serão afetadas, como comportamentos, regulação emocional e funções cognitivas (como funções executivas, memória, atenção, entre outras) (Nakayama et al., 2020).

Com isso, o objetivo deste artigo foi revisar na literatura, estudos atualizados sobre as consequências da exposição à maus-tratos na infância no desenvolvimento cerebral, tanto estruturais/anatômicas quanto funcionais.

\section{Método}

Foram revisados artigos na língua inglesa e portuguesa brasileira nas bases de dados Pubmed ( $n=358)$, Scielo ( $n=2)$ e Google Acadêmico ( $n=278)$, dos últimos 5 anos, através dos indexadores: early life stress, estresse precoce, maus-tratos na infância, childhood maltreatment e neurodesenvolvimento, e trauma na infância. A partir da análise dos resumos, foram excluídos artigos 
referentes a outras temáticas que não incluíssem aspectos relacionados à exposição ao trauma na infância e ao neurodesenvolvimento e funções cognitivas. Foram incluídos artigos de revisão e empíricos. Após exclusão, foram selecionados 23 artigos para compor o estudo de revisão aqui apresentado.

\section{Resultados}

Os resultados apresentados a seguir foram divididos em tópicos conforme os objetivos deste artigo, são eles: maus-tratos na infância e desenvolvimento cerebral e maus-tratos na infância e funcionamento cognitivo.

\section{Maus-tratos na infância e desenvolvimento cerebral}

Maus-tratos na infância promovem alterações na estrutura cerebral, na função e na conectividade, principalmente do circuito fronto-límbico e suas conexões. Além disso, gera mudanças fisiológicas, neuroquímicas e hormonais (Hart et al., 2017). As experiências na vida precoce produzem muitos efeitos na estrutura e no funcionamento do sistema nervoso central que se expressa de forma fidedigna na idade adulta (Ershov et al., 2018; Sarabdjitsingh, Loi, Joëls, Dijkhuizen, \&Van DerToorn, 2017). A separação materna prolongada e/ ou a negligência nos cuidados / maus tratos por terceiros ativam intensamente os sistemas de resposta ao estresse (Insana, Banihashemi, \& Germain, 2016). Esse estresse presente durante o desenvolvimento, ativa a resposta ao estresse, modificando a estrutura neurobiológica.

Algumas situações traumatizantes ficam registradas em nosso cérebro, na região subcortical, e geram mudanças nas estruturas e no funcionamento cerebral saudável, danificando e comprometendo a cognição (Thomason \& Marusak, 2017). Alguns estudos mostram alteração da estrutura de receptores gaba na amígdala. Esses receptores reagem ao ácido gama-aminobutírico (gaba), o principal neurotransmissor inibidor do cérebro, que atenua a excitabilidade elétrica dos neurônios (Fareri \& Tottenham, 2016; Grassi-0liveira, Honeycutt, Holland, Ganguly, \& Brenhouse, 2016). A redução da função desse neurotransmissor produz atividade elétrica excessiva. Tal descoberta proporcionou uma explicação molecular para constatação de anormalidades em eletroencefalogramas e irritabilidade límbica em pacientes que sofreram abuso na infância. Em um primeiro estágio ocorre a ativação dos sistemas de resposta ao estresse, ocorrendo o aumento dos níveis de cortisol, aumento da liberação de noradrenalina, aumento da tensão e da atenção e reações autonômicas (Insana et al., 2016).

Há consequências duradouras que incluem o desenvolvimento minimizado do hemisfério esquerdo, redução da integração entre os hemisférios cerebrais, aumento da irritabilidade elétrica dentro dos circuitos do sistema límbico (relacionado à emoção)(Insana et al., 2016). Existem consequências neuropsiquiátricas e vulnerabilidades a partir dessas alterações, que levam ao aumento do risco de desenvolver transtorno de estresse pós-traumático, depressão, transtorno de personalidade borderline, transtorno dissociativo e a uso nocivo de substâncias psicoativas (McCrory et al., 2017; Nemeroff, 2016; Puetz et al., 2017).

Os maus-tratos na infância podem alterar o córtex pré-frontal além de estimular o sistema límbico principalmente a amígdala a um estado de irritabilidade elétrica e químicas altas ou ainda danificar o hipocampo em desenvolvimento por meio de uma exposição excessiva aos hormônios do estresse devido à atividade no vermis cerebelar (Humphreys et al., 2020; Insana et al., 2016). Para qualquer nível de sintomatologia límbica, no entanto, a quantidade de fluxo sanguíneo no vermis cerebelar foi marcadamente menor em indivíduos com histórico de traumas.

A avaliação cerebral de crianças vítimas de abuso ou outra experiência emocional traumática apresentou alterações neurobiológicas semelhantes às encontradas em indivíduos que sofrem de transtornos mentais como a depressão: as mudanças estruturais e funcionais se davam nas mesmas regiões cerebrais (Puetz et al., 2017). Atividades de EEG (eletroencefalograma) anormais no lobo temporal também são frequentemente observadas em pessoas com comportamento autodestrutivo e forte propensão para o suicídio. Alguns transtornos mentais podem estar relacionados com uma disfunção no córtex pré-frontal e sua capacidade de modular estruturas límbicas tais como amígdala (fatores que tenham levado ao estabelecimento de um hiperfuncionamento de estruturas pré-corticais (como estruturas límbicas) (Bick \& Nelson, 2015). Experiências de maus tratos infantis têm sido associadas a elevados níveis de depressão, ansiedade e outros transtornos mentais, em função das alterações, ocorridas em longo prazo, em sistemas de neurotransmissores ligados à etiologia da depressão, como a serotonina (Fogelman \& Canli, 2019; Nemeroff, 2016; Queirós \& Caseiro, 2018).

Marcadores hormonais de resposta emocional (ACTH - Hormônio e CRF - Fator de liberação de corticotropina, além de concentração de cortisol) e taxa cardíaca em pacientes psiquiátricos e controles diante de uma tarefa ansiogênica. Em uma pesquisa, participantes foram designados em quatro grupos: pacientes deprimidos com ou sem história de abuso infantil e indivíduos sem diagnóstico psiquiátrico que sofreram e não sofreram este estresse precoce. Descobriu-se que altas taxas de ACTH (Hormônio adrenocorticotrófico) e cortisol e maior frequência cardíaca foram encontradas nos pacientes com história de estresse precoce (Agorastos et al., 2019). Os níveis de ACTH nas mulheres deprimidas com história de abuso foi seis vezes maior do que não sofreram maus tratos evidenciando a hiper-reatividade do eixo HPA. A maior reatividade do eixo HPA neste grupo sugere que as experiências precoces aversivas produzem efeitos duradouros no eixo HPA (Agorastos et al., 2019; Fogelman \& (anli, 2019). Além disso, as taxas cardíacas elevadas destes pacientes apontam também para uma maior ativação no sistema nervoso autonômico.

As áreas cerebrais que parecem estar envolvidas em síndromes de estresse traumático são a amígdala, o córtex pré-frontal medial, o giro cingulado anterior, o hipocampo, a ínsula e o córtex órbito-frontal. Essas áreas foram vistas como base em estudos que utilizam a neuroimagem em indivíduos com transtorno do estresse pós-traumático (TEPT) (Teicher, Andersen, Polcari, Anderson, \& Navalta, 2002). A neuroimagem - por meio da tomografia de emissão de pósitrons (TEP) - mostrou também mudanças de fluxo sanguíneo cerebral associado ao TEPT com maior ativação (aumento de fluxo) no hemisfério direito (córtex cingulado anterior e amígdala) e diminuição na área de broca, o que mostra coerência com a dificuldade de verbalização das experiências traumáticas (Borges \& Dell'Aglio, 2008). 0 abuso físico, sexual ou psicológico estava associado a anormalidades das ondas cerebrais em eletroencefalogramas (EEGs), que possibilitaram uma medida mais direta da irritabilidade límbica do que a checagem de que trabalhos subsequentes de outros pesquisadores, usando imageamento por ressonância magnética (IRM) confirmaram a associação entre maus tratos precoces e redução no tamanho do hipocampo e da amígdala do indivíduo adulto (Bick \& Nelson, 2015). 
Além de alterações estruturais, também há alterações na atividade neuronal no circuito fronto-límbico, que envolve o córtex frontal orbital esquerdo e o hipocampo esquerdo, regiões responsáveis pelo funcionamento executivo, regulação emocional, processos socioemocionais, funções autonômicas e regulação sono/vigília (Agorastos et al., 2019; Insana et al., 2016; Sarabdjitsingh et al., 2017).

A ocorrência de trauma infantil (decorrente de abuso físico, emocional ou sexual e negligência) tem sido associada à maior ativação do eixo hipotálamo hipófise- adrenal (HPA) A maior ativação deste eixo está relacionada ao aumento da resposta emocional frente a estímulos estressores (Thomason \& Marusak, 2017). Eventos traumáticos ocorridos durante o período de maior plasticidade neuronal geram uma supersensibilidade do sistema de resposta ao estresse, e de forma permanente (Penza et al. 2003).

\section{Maus-tratos na infância e funcionamento cognitivo}

As experiências traumáticas constituem fatores de risco para danos no desenvolvimento cognitivo a curto e longo prazo em crianças, adolescentes e adultos. A exposição a maus-tratos na infância torna as crianças mais suscetíveis a desenvolverem déficits em vários domínios do seu desenvolvimento (Thomason \& Marusak, 2017).

No nascimento, 0 cérebro ainda está em desenvolvimento, o qual sofre influência de fatores genéticos e ambientais. Os circuitos responsáveis pelas funções cognitivas mais refinadas também dependem dos fatores que operam em períodos específicos de desenvolvimento, modulando a função das áreas frontais responsáveis pelos pensamentos abstratos. A maturação cerebral e as funções cognitivas são sensíveis às experiências de vida, pois, elas irão afetar o desenvolvimento das estruturas e funções cerebrais subjacentes. Condições ambientais adequadas nas primeiras semanas de vida, associadas a uma estimulação eficiente com bom relacionamento materno são fundamentais para o desenvolvimento cognitivo e intelectual. Um ambiente pobre de estímulos pode ter influência direta no desenvolvimento motor desta criança, além de falta de concentração na escola, distúrbios de sono, atraso no desenvolvimento psicomotor, violência psicológica, tais como incapacidade de aprender, dificuldades escolares, hiperatividade e dificuldades de concentração (Thomason \& Marusak, 2017).

Para entender o impacto dos maus-tratos, principalmente a negligência, uma estratégia é observar o desenvolvimento de crianças que cresceram em instituições. Uma descoberta importante de tais estudos é que as crianças privadas de cuidados parentais e criadas em instituições apresentaram prejuízos em seu crescimento físico. Além disso, há algumas evidências de que crianças que são removidas de um ambiente adverso após sofrerem negligência e / ou abuso demonstram melhoria no desenvolvimento. Em geral, há um período sensível para recuperação do crescimento (1 ano de idade), que é mais curto que o período sensível para comprometimento do desenvolvimento (aproximadamente 2 anos). Há evidências de que um histórico de privação institucional está relacionado à menor desempenho cognitivo e acadêmico. Crianças carenciadas tendem a ter comprometimento cognitivo global duradouro, especialmente em termos de QI (quociente de inteligência). Outros estudos não encontraram associações significativas entre a duração da privação institucional dos 6 aos 42 meses de idade e os resultados cognitivos; no entanto, aos 11 anos, as crianças que sofreram menos de 6 meses de privação apresentaram menor QI verbal (Thomason \& Marusak, 2017) e baixos escores de compreensão de leitura. Em consonância com esses resultados, al guns estudos encontraram déficits no funcionamento intelectual e cognitivo de crianças maltratadas, em comparação com crianças que não sofreram abuso. Essas pesquisas constataram consistentemente que maus-tratos aumentam o risco de baixo desempenho acadêmico e desempenho escolar precário. Estudos em ratos demonstraram que uma separação maternal prolongada (3h por dia) gera quantidades significativas de estresse resultando em mudanças emocionais relacionadas ao comportamento, reatividade ao estresse e prejuízos nas funções cognitivas, principalmente na memória de longo prazo e no aprendizado (Dunn et al., 2017; Ershov et al., 2018; Sarabdjitsingh et al., 2017).

0 efeito do abuso infantil pode manifestar-se de várias formas, em qualquer idade. Internamente, pode aparecer como depressão, ansiedade, pensamentos suicidas ou estresse pós-traumático (Grassi-Oliveira et al., 2016; Queirós \& (aseiro, 2018); pode também expressar-se externamente como agressão, impulsividade, delinquência, hiperatividade ou abuso de substâncias (Ershov et al., 2018). Uma condição psiquiátrica fortemente associada a maus tratos na infância é o chamado distúrbio de personalidade limítrofe (transtorno de personalidade borderline). 0 indivíduo com essa disfunção tem como característica enxergar os outros em termos de preto ou branco, oito ou oitenta, muitas vezes colocando seus interlocutores num pedestal, para depois transformá-los em vilões, a partir de algo percebido como desfeita ou traição. Aqueles que sofrem desse transtorno são propensos a explosões de raiva e episódios transitórios de paranoia ou psicose. Eles possuem tipicamente uma história de relações intensas e instáveis (Ershov et al., 2018).

O EP parece estar associado com alterações nas funções cognitivas, entre elas: velocidade de processamento, funções executivas (Welsh et al., 2017), controle inibitório, processamento emocional e memória na vida adulta (Agorastos et al., 2019; Insana et al., 2016).

\section{Considerações finais}

Os efeitos de todo e qualquer abuso, maus tratos, negligências são devastadores, principalmente no início do desenvolvimento humano, onde as estruturas cerebrais também estão se desenvolvendo, acarretando alterações irreversíveis em sua estrutura física.

As negligências e maus tratos na primeira infância, assim como a partir do nascimento, já causam problemas no neurodesenvolvimento e na produção de uma futura cognição. Estudos relacionando influência genética e epigenética têm buscado compreender os fatores desencadeantes dos maus-tratos na infância e principalmente buscar compreender as variações individuais quanto à resiliência e vulnerabilidade ao estresse.

Os estudos seguem buscando compreender as consequências que os maus tratos na infância podem provocar, mas, tratando-se de prejuízos cognitivos, há evidências de que o abuso pode ocasionar déficits no controle emocional, na tomada de decisão, e prejuízos na memória de trabalho, além de desregular os níveis de cortisol, que também gera consequências emocionais e cognitivas. Além disso, os maus tratos na infância estão associados com redução do volume do hipocampo. Os maus tratos não provocam somente sofrimento psíquico e emocional, mas alteram a morfologia e fisiologia cerebral, principalmente o lóbulo pré-frontal, sistema límbico e, como vimos, também o corpo caloso. 
Um termo que vem se utilizando para casos de crianças que sofrem ou sofreram diversos traumas, é Trauma Complexo, que descreve as consequências da exposição contínua e prolongada a eventos estressores de natureza interpessoal. Além dos sintomas de TEPT, as crianças que vivenciam esses traumas durante seu desenvolvimento, apresentam outros sintomas, como: problemas na regulação emocional, drásticas mudanças de humor e no padrão comportamental, perda de autonomia, perda de regulação do sono, da fome e de autocuidados, múltiplos problemas somáticos, aumento dos comportamentos de risco, sentimentos de ódio, sentimento de culpa e sentimentos de impotência. Crianças expostas a contínuas vivências traumáticas de natureza interpessoal irão experimentar emoções intensas de raiva, medo, resignação, vergonha, sensação de derrota e traição. 0 menor estímulo seria capaz de desencadear medo intenso. Soma-se a isso o fato de que a expectativa de retorno do trauma permeia os relacionamentos das crianças vítimas de trauma complexo. Esse termo pode auxiliar e englobar as diversas consequências que esses eventos traumáticos precoces podem causar no desenvolvimento cerebral, emocional, cognitivo e social dessas crianças.

Uma limitação vista nos estudos foi quanto à dificuldade em se especificar diferenças individuais quanto a severidade e frequência da experiência de maus-tratos, e a idade em que ocorreu ou ocorreram os episódios. Essas variáveis podem representar diferentes desfechos e consequências neurodesenvolvimentais a partir da experiência traumática. Também foi visto que diferentes tipos de maus-tratos interagem de forma singular nos mecanismos neurobiológicos do indivíduo.

Contudo, tendo em vista o impacto social, físico, cognitivo e psicológico que os maus-tratos causam, é indispensável a atenção na sua prevenção, detecção precoce e intervenção, pois seus danos podem manter-se por toda vida.

\section{Referências}

Agorastos, A., Pervanidou, P., \& Chrousos, G. P. (2019). Developmental Trajectories of Early Life Stress and Trauma: A Narrative Review on Neurobiological Aspects Beyond Stress System Dysregulation, 10(March), 1-25. https:// doi.org/10.3389/fpsyt.2019.00118

Bath, K. G., Manzano-Nieves, G., \& Goodwill, H. (2016). Early life stress accelerates behavioral and neural maturation of the hippocampus in male mice. Hormones and Behavior, 82,64-71. https://doi.org/10.1016/j.yhbeh.2016.04.010

Bick, J., \& Nelson, C. A. (2015). Early Adverse Experiences and the Developing Brain, 41(1), 177-196. https://doi.org/10.1038/npp.2015.252

Borges, J. L., \& Dell'Aglio, D. D. (2008). Relações entre abuso sexual na infância, transtorno de estresse pós-traumático (TEPT) e prejuízos cognitivos. Psicologia em Estudo, 13(2), 371-379. https://doi.org/10.1590/S141373722008000200020

Dunn, E. C., Busso, D. S., Raffeld, M. R., Jordan, W., Nelson, C. A., Doyle, A. E., \& Luk, G. (2017). HHS Public Access, 181-191. https://doi.org/10.1016/j. chiabu.2015.10.014.Does

Ershov, N. I., Bondar, N. P., Lepeshko, A. A., Reshetnikov, V. V., Ryabushkina, J. A., \& Merkulova, T. I. (2018). Consequences of early life stress on genomic landscape of H3K4me3 in prefrontal cortex of adult mice. BMC Genomics, 19(Suppl 3). https://doi.org/10.1186/s12864-018-4479-2

Fareri, D. S., \& Tottenham, N. (2016). Effects of early life stress on amygdala and striatal development. Developmental Cognitive Neuroscience, 19, 233-247. https://doi.org/10.1016/j.den.2016.04.005

Fogelman, N., \& Canli, T. (2019). Early life stress, physiology, and genetics: A review. Frontiers in Psychology, 10(JULY). https://doi.org/10.3389/ fpsyg.2019.01668
Grassi-Oliveira, R., Honeycutt, J. A., Holland, F. H., Ganguly, P., \& Brenhouse, H. C. (2016). Cognitive impairment effects of early life stress in adolescents can be predicted with early biomarkers: Impacts of sex, experience, and cytokines. Psychoneuroendocrinology, 71, 19-30. https://doi.org/10.1016/j. psyneuen.2016.04.016

Harms, M. B., Shannon Bowen, K. E., Hanson, J. L., \& Pollak, S. D. (2018). Instrumental learning and cognitive flexibility processes are impaired in children exposed to early life stress. Developmental Science, 21(4), 1-13. https://doi.org/10.1111/desc. 12596

Hart, H., Lim, L., Mehta, M. A., Chatzieffraimidou, A., Curtis, C., Xu, X., ... Rubia, K. (2017). Reduced functional connectivity of fronto-parietal sustained attention networks in severe childhood abuse. PLOS ONE, 12(11), 1-17. https://doi.org/10.1371/journal.pone.0188744

Humphreys, K. L., King, L. S., Sacchet, M. D., Catalina, M., Colich, N. L., Ordaz, S. J., ... Gotlib, H. (2020). HHS Public Access, 22(3), 1-18. https://doi. org/10.1111/desc.12775.Evidence

Insana, S. P., Banihashemi, L., \& Germain, A. (2016). Childhood maltreatment is associated with altered frontolimbic neurobiological activity during wakefulness in adulthood, 28(2), 551-564. https://doi.org/10.1017/ S0954579415000589.Childhood

McCrory, E. J., Gerin, M. I., \& Viding, E. (2017). Annual Research Review: Childhood maltreatment, latent vulnerability and the shift to preventative psychiatry - the contribution of functional brain imaging. Journal of Child Psychology and Psychiatry and Allied Disciplines, 58(4), 338-357. https:// doi.org/10.1111/jcpp.12713

Nakayama, M., Hori, H., Itoh, M., Lin, M., Niwa, M., Ino, K., ... Kim, Y. (2020). Possible Long-Term Effects of Childhood Maltreatment on Cognitive Function in Adult Women With Posttraumatic Stress Disorder. Frontiers in Psychiatry, 11(April), 1-10. https://doi.org/10.3389/fpsyt.2020.00344

Nemeroff, C. B. (2016). Review Paradise Lost : The Neurobiological and Clinical Consequences of Child Abuse and Neglect. Neuron, 89(5), 892-909. https://doi.org/10.1016/j.neuron.2016.01.019

Puetz, V. B., Parker, D., Kohn, N., Dahmen, B., Verma, R., \& Konrad, K. (2017). Altered brain network integrity after childhood maltreatment: A structural connectomic DTI-study. Human Brain Mapping, 38(2), 855-868. https:// doi.org/10.1002/hbm.23423

Queirós, M., \& Caseiro, J. (2018). Child maltreatment and mental disorders: the role of epigenetics. Nascer e Crescer, 27(3), 166-175. https://doi. org/10.25753/BirthGrowthMJ.v27.i3.13431

Sarabdjitsingh, R. A., Loi, M., Joëls, M., Dijkhuizen, R. M., \& Van Der Toorn, A. (2017). Early life stress-induced alterations in rat brain structures measured with high resolution MRI. PLOSONE, 12(9), 1-14. https://doi.org/10.1371/ journal.pone.0185061

Teicher; "M. H., \& Samson1., J. A. (2016). Enduring neurobiological effects of childhood abuse and neglect. J Child Psychol Psychiatry, 57(3), 241-266. https://doi.org/10.1111/jcpp.12507.Annual

Teicher, M. H., Andersen, S. L., Polcari, A., Anderson, C. M., \& Navalta, C. P. (2002). Developmental neurobiology of childhood stress and trauma, 25, 397-426.

Thomason, M. E., \& Marusak, H. A. (2017). REVIEWTOWARD UNDERSTANDING THE IMPACT OF TRAUMA ON THE. Neuroscience, 342, 55-67. https://doi. org/10.1016/j.neuroscience.2016.02.022

Welsh, M. C., Peterson, E., \& Jameson, M. M. (2017). History of Childhood Maltreatment and College Academic Outcomes : Indirect Effects of Hot Execution Function, 8(July), 1-13. https://doi.org/10.3389/fpsyg.2017.01091 